\title{
Die großen wissenschaftlichen Leistungen von Stefan Vogel (1925-2015) Teil 5c. Öl statt Nektar - die Ölblumen (monokotyle Vertreter und abschließende Bemerkungen)
}

\author{
Anton Weber, Günter Gerlach \& Hanno Schaefer
}

\begin{abstract}
In Monocotyledons, oil flowers occur in two families: Iridaceae and Orchidaceae. Iridaceae oil flowers evolved two times (with trichome elaiophors), in the latter at least 10 times independently (with both trichome- and epithelial elaiophores). Significant representatives of the two families, discovered by STEFAN Vogel, are described and illustrated. We conclude with final remarks on biogeography of oil flowers, evolution and loss of floral oil production, mimicry, and prediction in science.
\end{abstract}

\section{Zusammenfassung}

Bei den Monokotyledonen haben sich Ölblumen in zwei Familien entwickelt: Iridaceen und Orchideen. Bei ersteren (mit Trichom-Elaiophoren) zweimal, bei letzteren mindestens 10 mal voneinander unabhängig (sowohl Trichom- als auch Epithel-Elaiophoren). Wichtige Vertreter aus beiden Familien, die bereits von STEFAN VogEL entdeckt wurden, werden näher dargestellt und illustriert. Abschließend werden folgende Aspekte der Ölblumen angesprochen: Pflanzengeographie, Evolution und sekundärer Verlust der floralen Ölproduktion, Mimikry und Voraussage in der Wissenschaft.

\section{Einleitung}

Außer bei den Dikotyledonen, für die wir das Auftreten von Ölblumen beschreiben konnten (vgl. Dötterl, Gerlach \& Weber 2018), sind es bei den Monokotyledonen nur zwei: die Iridaceae und die Orchidaceae. Allerding enthalten letztere fast so viele ölblütige Arten wie alle Dikotyledonen zusammen. Die Entdeckung der Ölblumen bei den Irisgewächsen und den Orchideen geht auf $S_{\mathrm{TE}}-$ FAN Vogel zurück, der die beiden Familien bereits im ersten Band der „Ölblumen-Trilogie“ (VogeL 1974) ausführlich behandelt hat. Im Folgenden werden die Ölblumen der beiden Familien näher vorgestellt. Daran schließen sich allgemeine und zusammenfassende Bemerkungen über die Ölblumen insgesamt an.

\section{Iridaceae: Cypella, Ennealophus, Trimezia, Tritoniopsis}

Die Verbreitung von Ölblumen bei den Iridaceae ist mit einer erst viel später entdeckten Ausnahme (Tritoniopsis parviflora) auf Südamerika beschränkt. Es handelt sich um Arten aus sieben Gattungen (Alophia, Cypella, Ennealophus, Gelasine, Sisyrinchium, Tigridia, Trimezia, Tritoniopsis), von denen hier nur die in der Kapitel-Überschrift genannten Gattungen behandelt werden. Diese spielen auch als Zierpflanzen eine gewisse Rolle. Alle von Stefan Vogel gemachten Beobachtungen zur Bestäubung von Iridaceae kamen im Norden Argentiniens zu Stande. Es handelt sich bei den Pflanzen um Knollengeophyten, bei denen das Öl in Trichom-Elaiophoren gebildet und präsentiert wird.

Die Blüten von Ennealophus euryandrus (von Vogel 1974 unter dem Namen Sphenostigma euryandra abgehandelt) haben wie viele andere Iridaceae drei große äußere und drei kleine innere Tepalen. Letztere sind im unteren, breiteren Teil geknickt, wobei die basalen Teile einen schüssel- oder glockenförmigen Raum bilden, während die distalen, schmäleren Teile zurückgeschlagen sind. Dadurch kommt eine Art Separatkrone zustande. Der untere Teil des schüsselförmigen Raumes ist dicht mit ölsezernierenden Trichomen besetzt. Als Bestäuber konnte Vogel Bienen der Gattung Paratetrapedia feststellen. Diese müssen eine schiefe Haltung einnehmen und sich in den Blütenbecher hineinbeugen, um das begehrte Öl aufsammeln zu können. Sie schaben mit ihren besonders ausgebildeten Vorderbeinen das Öl aus den Trichomfluren und überführen es in die nach oben gestreckten hinteren Sammelbeine. Beim Sammeln umwandern die Tiere die zentrale Staubblattsäule und kommen dabei mit den Antheren und den Narben in Berührung.

Ähnlich wie bei den Schwertlilien (Iris) sind die Blüten von Cypella und Trimezia in drei funk- 


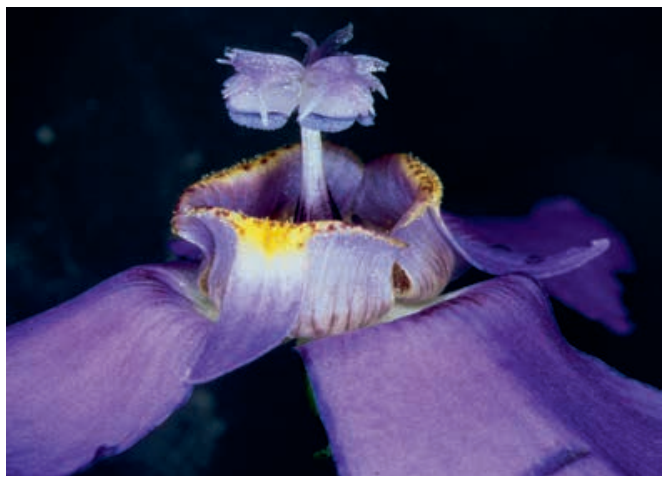

Abb. 1a: Ennealophus euryandrus (Iridaceae). Blüte in Seitenansicht. (Foto: G. Gerlach, Bolivien, Dept. Santa Cruz, Jagüe, 2140m; cult. BG München-Nymphenburg).

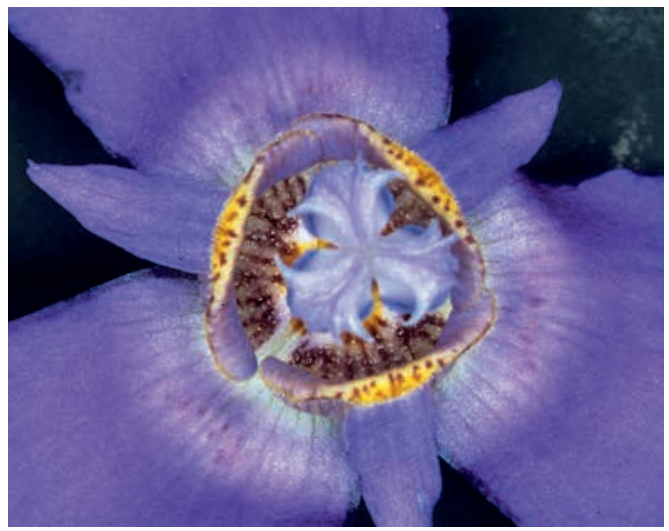

Abb. 1b: Ennealophus euryandrus (Iridaceae). Blüte von oben gesehen. (Foto: G. Gerlach, Bolivien, Dept. Santa Cruz, Jagüe, 2140m; cult. BG München-Nymphenburg).

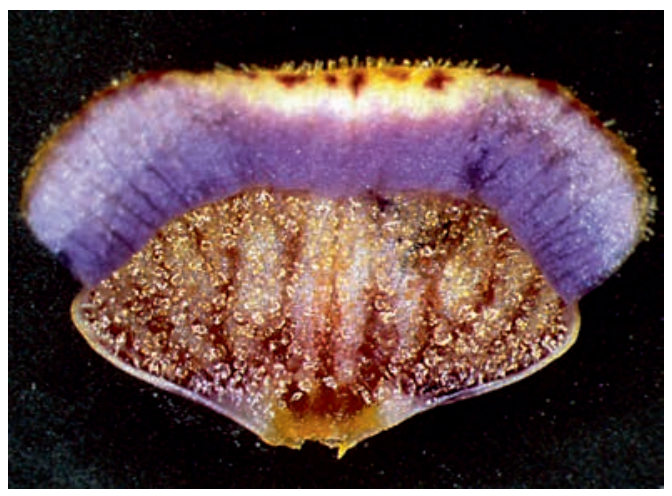

Abb. 1c: Ennealophus euryandrus (Iridaceae). Unterer Teil eines inneren Tepalums, mit Öldrüsen bzw. Ölfilm an der Basis. (Foto: G. Gerlach, Bolivien, Dept. Santa Cruz, Jagüe, 2140m; cult. BG München-Nymphenburg).

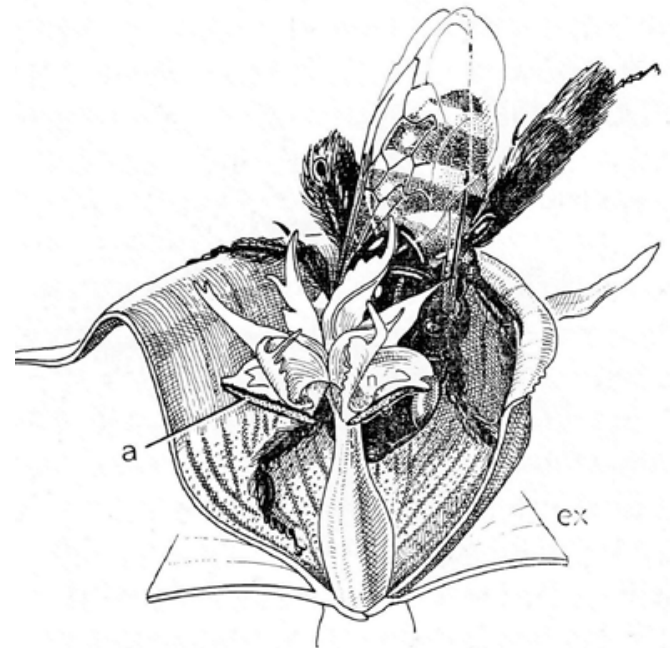

Abb. 2. Ennealophus euryandrus (Iridaceae); innerer Blütenteil (becherförmige Separatkrone) mit Biene (Paratetrapedia melampoda) beim Aufsammeln des Blütenöls mit den Vorderbeinen (aus Vogel 1974, Abb. 59).

tionelle Einheiten (Meranthien) gegliedert. Entsprechend sind auch die ölabsondernden Trichome auf die drei Einheiten aufgeteilt. Somit stehen hier den Bestäubern drei Zugänge zu den Ölressourcen zur Verfügung und der Bestäubungsvorgang sieht gänzlich anders aus: Die Bienen (Arten der Gattungen Centris und Chalepogenus) landen auf den lippenartigen inneren Tepalen, die an den Basen die Elaiophoren tragen und durch ein $\mathrm{Ge}-$ lenk beweglich sind. Beim Vordringen zu den basalen Elaiophoren kippen die Tepalen gegen die Staubblattsäule und die Narben, wodurch die Bienen mit Pollen beladen werden bzw. den mitgebrachten Pollen abladen.

Erst 2002 wurde mit Tritoniopsis parviflora ein erstes ölanbietendes Irisgewächs außerhalb Südamerikas, nämlich in Südafrika, gefunden (MANNing et al. 2002, MANning \& Goldblatt 2005, Goldblatt \& Manning 2006), und zwar einer einzigen Art der Gattung Tritoniopsis. Sie wird von Rediviva gigas bestäubt, also einer Art jener Bienengattung, die für die Bestäubung von Diascia (Scrophulariaceae) zuständig ist (vgl. DötTerL, Gerlach \& Weber 2018)). Da Tritoniopsis in einen völlig anderen Verwandtschaftskreis (Unterfam. Crocoideae) als die südamerikanischen Iridaceae (alle Unterfam. Iridoideae) gehört, ist es 


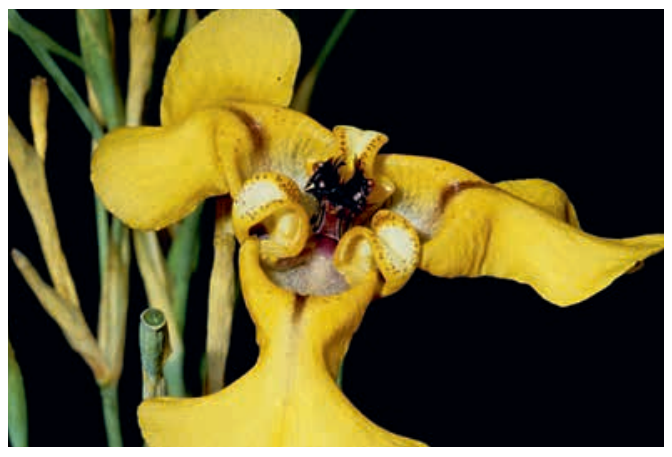

Abb. 3a: Cypella herbertii (Iridaceae); sie Iris-artige Blüte hat drei kleine, bewegliche, innere Tepalen, auf deren Mittelrippe die Elaiophoren gelegen sind. (Fotos G. Gerlach, BG München-Nymphenburg).

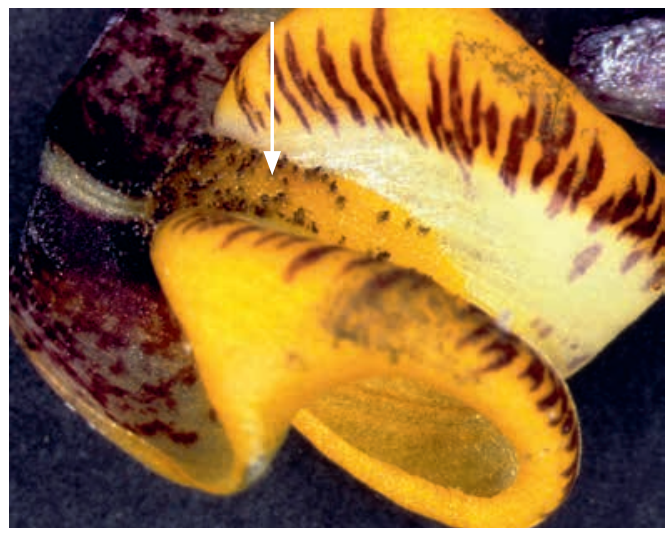

Abb. 3b: Cypella herbertii (Iridaceae); mit einem Pfeil sind die Elaiophoren markiert. (Foto: G. Gerlach, BG München-Nymphenburg).

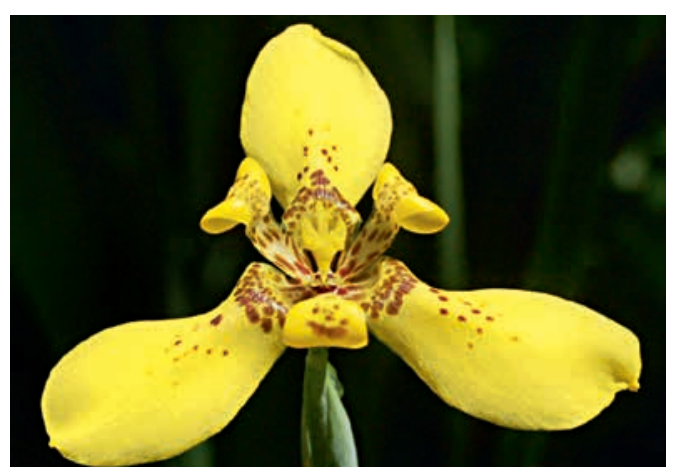

Abb. 4: Trimezia martinicensis und (im Bild) T. steyermarkii (Iridaceae) sind in den Tropen der ganzen Welt häufig kultivierte Zierpflanzen. Ihre Blüten funktionieren ähnlich wie jene von Cypella (Foto: A. Weber; Costa Rica, Tropenstation La Gamba).

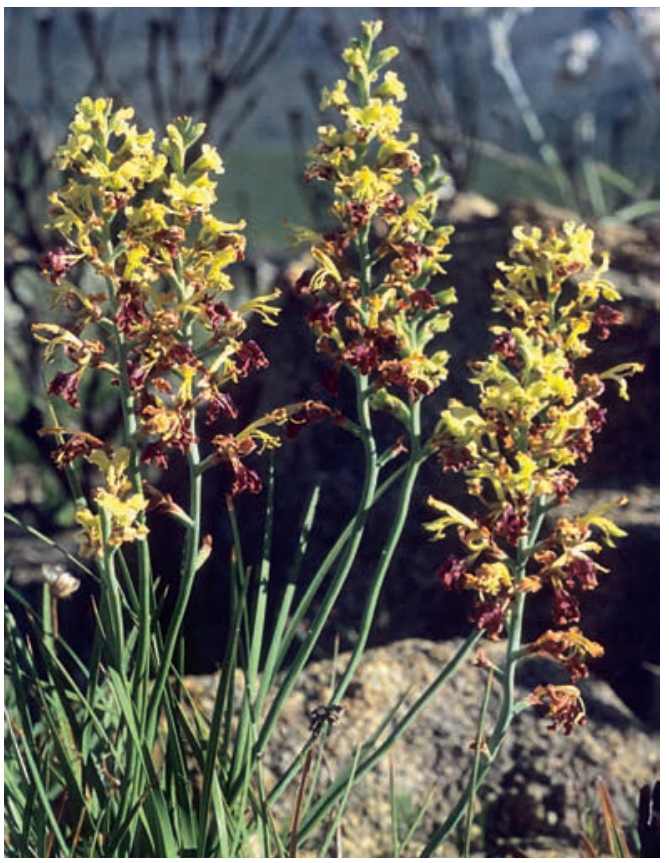

Abb. 5: Tritoniopsis parviflora (Iridaceae). Diese Art ist das einzige Irisgewächs außerhalb der Neotropen bzw. in Südafrika, deren Blüten Öl anbieten (Foto: J. MannING; Südafrika, Fuß des St. Lowry's Passes).

augenscheinlich, dass innerhalb der Familie das Ölblumensyndrom mindestens zweimal unabhängig entstanden sein muss. Das ist auch daraus ersichtlich, dass Tritoniopsis einen Epithel-Elaiophor hat, der sich an der Basis der Tepalen und im Schlund der kurzröhrigen Blüte befindet.

\section{Orchideen dürfen natürlich nicht fehlen}

Wenn es um blütenökologische Besonderheiten geht, kann man fast sicher sein, dass die Orchideen mit dabei sind. Tatsächlich entwickelt eine Vielzahl von Orchideen ölanbietende Blüten. Diese sind in der Familie mehrmals parallel entstanden. Es haben sich, wie bereits Vogel (1974) feststellen konnte, unabhängig in verschiedenen Verwandtschaftskreisen sowohl Epithel- als auch Trichom-Elaiophoren entwickelt. Als Beispiele für Orchideen mit Trichom-Elaiophoren können die Gattungen Zygostates, Ornithocephalus und Lockhartia genannt werden, die Mehrzahl der ölblütigen Orchideen bringt hingegen Epithel-Elaiophoren hervor (Oncidium p.p., Gomesa p.p.). Auch bezüglich der pflanzengeographischen Verbreitung 
sind zwei große Gruppen von öldarbietenden Orchideen zu unterscheiden: die neotropischen und die südafrikanischen Vertreter. Letztere wurden nicht von Vogel, sondern in den 1980er Jahren von südafrikanischen Forschern entdeckt.

Was die Bestäubung betrifft, konnte VoGEL selbst keine Beobachtungen machen, und das ist bis heute so geblieben: bis dato liegen keine eingehenden Studien zur Bestäubung ölanbietender Orchideen vor. Gut in das Bild passen ältere Beobachtungen des amerikanischen Orchideenforschers C. H. Dodson (in van der Pijl \& Dodson 1966). Er konnte über Jahre in Iquitos (Peru) dokumentieren, dass manche Orchideen von Bienen der Gattung Paratetrapedia (Apidae-Apinae-Tapinotaspidini) besucht werden, deren Weibchen, wie wir heute wissen, Blütenöl sammeln.

Praktisch alles, was bezüglich der Bestäubung von ölanbietenden Orchideen bekannt ist, bzw. angenommen werden kann, geht auf Vogels Rückschlüsse aus seinen morphologischen Analysen zurück.

Von den Orchideen mit Trichom-Elaiophoren hat Vogel (1974) eine Art der Gattung Zygostates (Z. pustulata) eingehend analysiert. Die Blüten

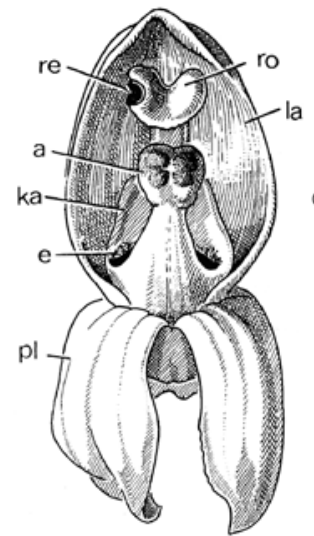

A

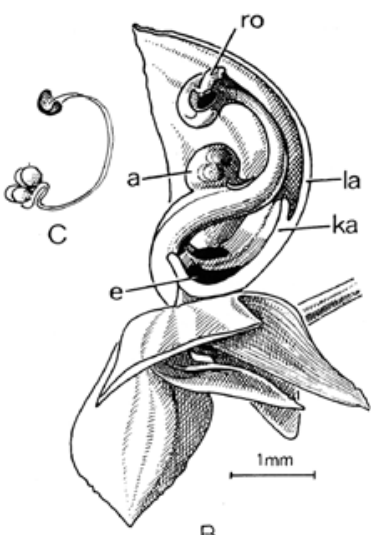

B
Abb. 6: Zygostates pustulata (Orchidaceae), Blüte mit Trichom-Elaiophoren. (A) Vorderansicht, (B) medianparalleler Schnitt. Die beiden Elaiophoren (e) liegen seitlich an der Basis des Lippenkallus (ka) und stellen Fluren aus einzelligen, schlauchförmigen Drüsenhaaren dar (aus VogeL 1974, Abb. 71). sind so orientiert, dass das Labellum nach oben zeigt. Es sind zwei Elaiophoren vorhanden, die seitlich an der Basis des Lippenkallus liegen und aus einzelligen, schlauchförmigen Drüsenhaaren bestehen.

Die Arten von Ornithocephalus und verwandter Gattungen haben sehr kleine, oft weißlich-grünlich oder gelblich gefärbte Blüten, die zu dichten Blütenständen vereinigt sind. Sie tragen eine Säule mit einem langen, schnabelförmigen Fortsatz (Rostellum) und bilden Pollinarien mit einem extrem langen Stielchen. An der Basis der Lippe sitzt ein auffälliger Elaiophor, der üppig mit Öl gefüllt ist und dem Epithel-Typus ähnelt. Saugt man das Öl aber mit Filterpapier ab, so treten dichte Trichomfelder zu Tage. Wie schon Dodson (in VAN Der Pijl \& Dodson 1966) berichtet hat, werden die Blüten von Tapinotaspidinen ausgebeutet. Stefan Vogel hat viele Arten dieser Gruppe ausgiebig in ihrer Blütenmorphologie untersucht. Auf der Tagung der Deutschen Botanischen Gesellschaft 2010 in Wien präsentierte Vogel ein Poster (in englischer Version), in dem die winzigen, öldarbietenden Blüten von zahlreichen Arten von Ornithocephalus und verwandter Gattungen in handkolorierten Zeichnungen dargestellt sind ein Meisterstück botanischer Illustration (deutsche Version siehe Abb. 8).

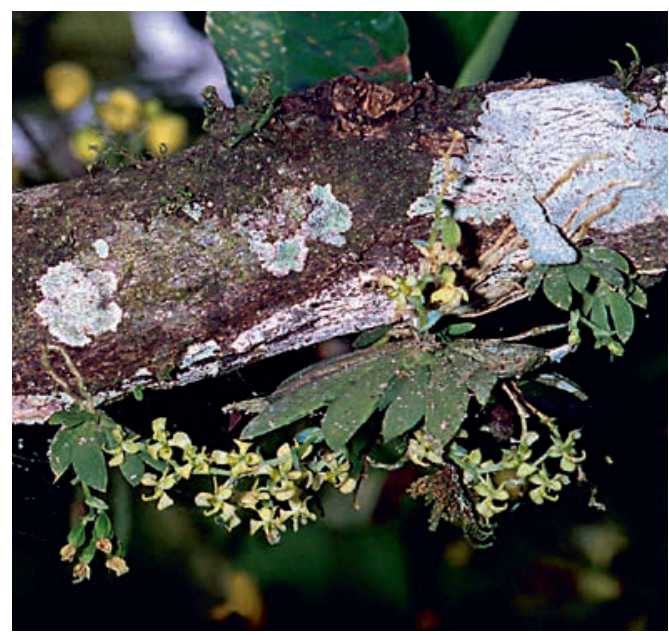

Abb. 7: Ornithocephalus falcatus (Orchidaceae), ein wenige Zentimeter großer Epiphyt mit winzigen Blüten an der Unterseite eines Baumastes (Foto: G. Gerlach; Ecuador, Prov. Morona Santiago, Gualaquiza). 

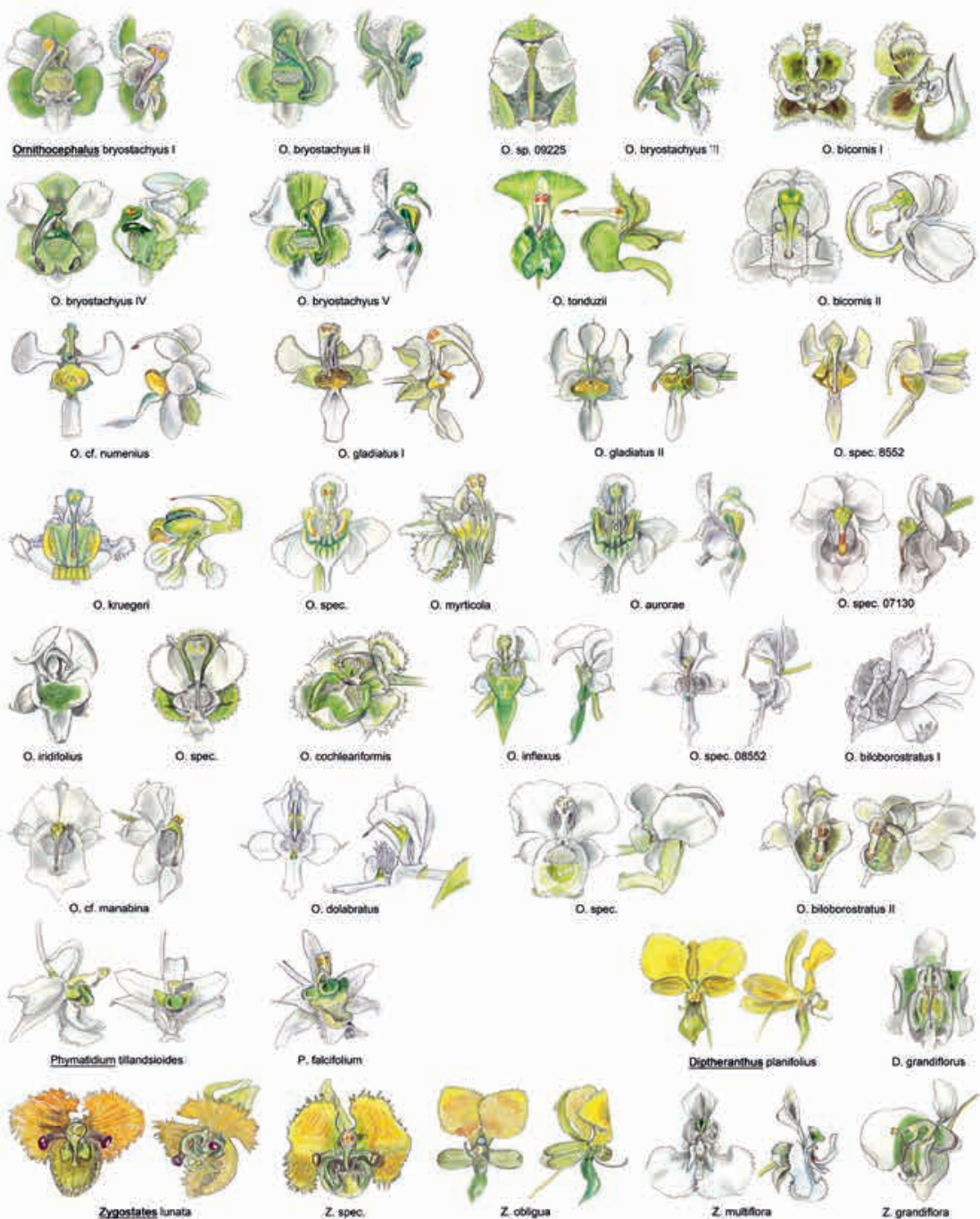

zrostates lunata

z. spec.
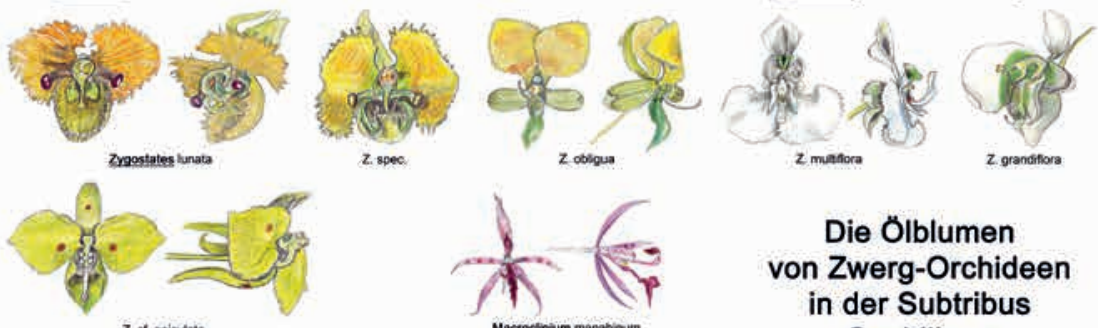

Zot apiadata
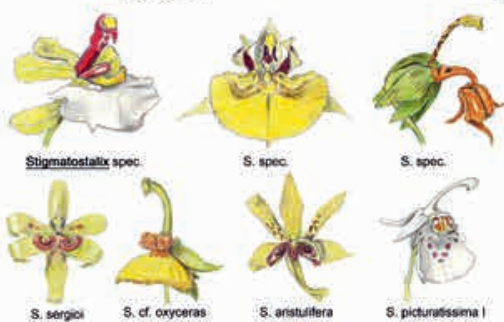
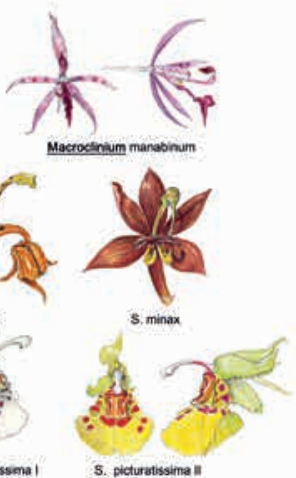

\section{Die Ölblumen von Zwerg-Orchideen in der Subtribus Oncidiinae}

\section{Ein ikonographischer Öberblick}

von

\section{Prof. Dr. Stefan Vogel}

Zentrum fur Biodiversitat der Universitat Wien

Abb. 8: Poster von Stefan Vogel mit kolorierten Handzeichnungen verschiedener ölblütiger Arten der Subtribus Oncidiinae (Orchidaceae) aus Lateinamerika (lediglich das rosafarbige Macroclinium ist nicht ölblütig, sondern gehört zu den Parfümblumen). Eine englische Version des Posters wurde anlässlich einer Tagung der Sektion „Biodiversität und Evolutionsbiologie“ der Deutschen Botanischen Gesellschaft in Wien, 16.-19. Sept. 2010, präsentiert. 
Abgesehen von den Angaben Dodsons gibt es keine weiteren Beobachtungen zu ihrer Bestäubungsbiologie. Dem Zweitautor dieses Beitrags liegen einige Prachtbienenmännchen mit Ornithocephalus-Pollinarien vor. Es ist aber wahrscheinlich, dass die Prachtbienen nur Zufallsbesucher sind und ölsammelnde Bienen (Tapinotaspidinen?) die legitimen Bestäuber darstellen.

Von den Orchideen-Gattungen mit Epithel-Elaiophoren ist an erster Stelle die große Gattung Oncidium zu nennen. Die große Mehrzahl der Oncidium-Arten entwickeln gelbe Blüten. Davon sind zahlreiche, aber keineswegs alle, Arten ölblütig. Neben dieser Tatsache ist die Ähnlichkeit der Blüten mit jenen der Malpighiaceen in Größe, Form und Färbung (gelb, seltener rosa) auffällig. Es ist klar, dass die ölführenden Oncidium-Blüten wie jene der Malpighiaceen von ölsammelnden Bienen bestäubt werden müssen, doch gibt es dazu keine publizierten Beobachtungen. Üblicherweise haben die ölblütigen Arten der Oncidiinae zwei Elaiophoren. Sie sind auf den Schultern der Lippe und hinter der Blütenebene gelegen. Die morpho-
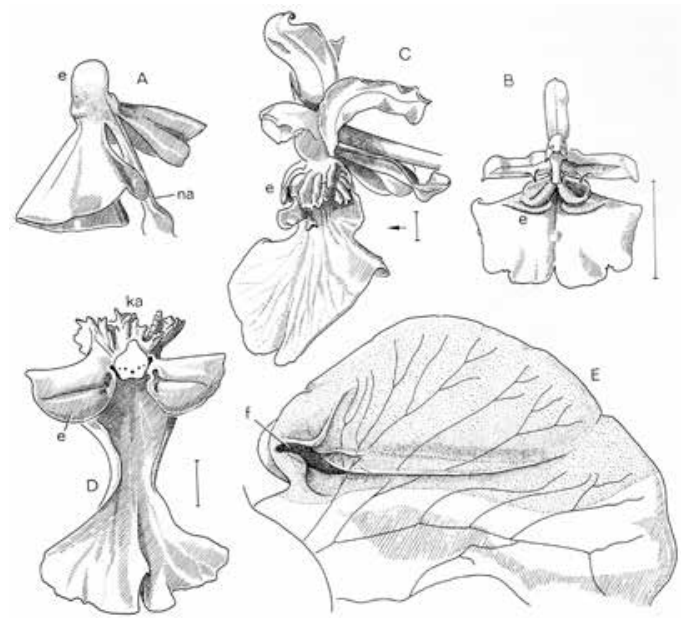

Abb. 9: Orchideen mit Epithel-Elaiophoren (e). A - C Arten mit Kallusdrüsen: A Oncidium bicallosoides (bei Vogel als Sigmatostalix bicallosa), Labellum; B Oncidium gramineum (bei Vogel als Sigmatostalix graminea), ganze Blüte; C Gomesa viperina (bei Vogel als Oncidium viperinum), ganze Blüte; D Oncidium sotoanum (bei Vogel als Oncidium ornithorhynchum), Rückenansicht des Labellums mit drüsigen Auriculae und nicht-drüsigem Kallus (ka); E Auricula vergrößert, das Öl wird von der punktierten Fläche und in der Furche (f) abgesondert (aus Vogel 1974, Abb. 30). logische Anpassung der Blüten von Oncidium exalatum, O. iricolor und O. luteum an ölsammelnde Bienen geht sogar so weit, dass auf der Lippe vier Öldrüsen ausgebildet sind. Da diejenigen Bienen, die Malpighiaceen-Blüten ausbeuten, mit vier Beinen sammeln, liegt hier offensichtlich ein Fall von Mimikry (Nachahmung von Malpighiaceen-Blüten) vor, vgl. dazu auch die Ausführungen in den abschließenden Bemerkungen weiter unten.

Deutlich mehr als die Hälfte aller Oncidium-Arten haben allerdings Blüten, die weder Öl noch Nektar noch sonst etwas darbieten. Hier ähneln glänzende Bereiche der Lippenoberfläche fett-glänzenden Elaiophoren. Es handelt sich um Täuschblumen, genauer gesagt um Öltäuschblumen. Sie profitieren davon, dass die botanisch ungebildeten Bienen sie schlicht mit ölführenden $\mathrm{On}$ cidium- oder Malpighiaceen-Blüten verwechseln.

Alle Arten der ehemaligen Gattung Sigmatostalix (heute in Oncidium eingegliedert) und Gomesa radicans (bei Vogel als Ornithophora radicans) bilden ebenfalls auffällige Epithel-Elaiophoren, die oft in

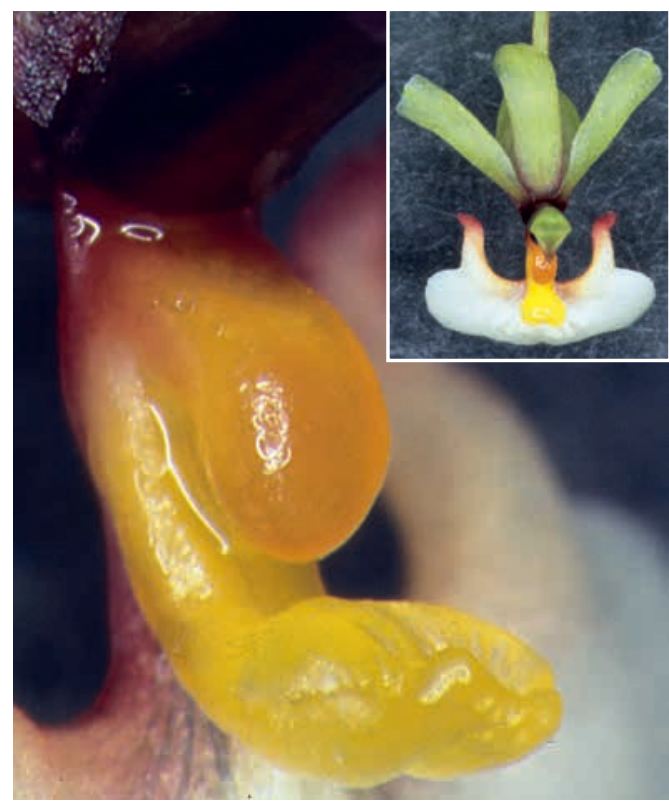

Abb. 10: Gomesa radicans (bei Vogel Ornithophora radicans, Orchidaceae). Die orange und gelb gefärbten Schwielen (Calli) an der Basis des Labellums sind zu einem Epithel-Elaiophor umgewandelt (Foto: G. GerLach, BG München-Nymphenburg). 
schalenförmigen Strukturen zu finden sind. VoGEL (1974) untersuchte die Morphologie von O. bicallosoides (bei Vogel als Sigmatostalix bicallosa) und schloss daraus auf den Ablauf der Bestäubung. Bis heute gibt es leider keine Beobachtungen dazu.

Weiterhin zeigt auch Lockhartia, ebenfalls aus der Verwandtschaft von Oncidium, Trichom-Elaiophoren. Die Pflanzen sind wenig- und kleinblütig und treten nie in größeren Populationen auf. Es ist schwer vorstellbar, dass die Bestäuber aus dem Blütenöl dieser Pflanzen ihre Nachkommenschaft mit Futtervorräten versorgen können. Ob hier Mimikry-Verhältnisse nach dem Muster von $O n$ cidium vorliegen, ist bis dato ungeklärt.

Den mehr als 200 neotropischen Orchideenarten mit Ölblumensyndrom steht etwa die halbe Anzahl an Öl anbietenden Arten aus dem südlichen Afrika gegenüber. Dabei handelt es sich durchwegs um terrestrische Orchideen aus der Unterfamilie Orchidoideae, Tribus Diseae (jene der Neuen Welt sind ausschließlich Epiphyten und gehören der Unterfamilie der Epidendroideae an).

Schon zu Beginn seiner wissenschaftlichen Karriere beschäftigte sich STEFAN Vogel intensiv mit diesen südafrikanischen Orchideen (Vogel

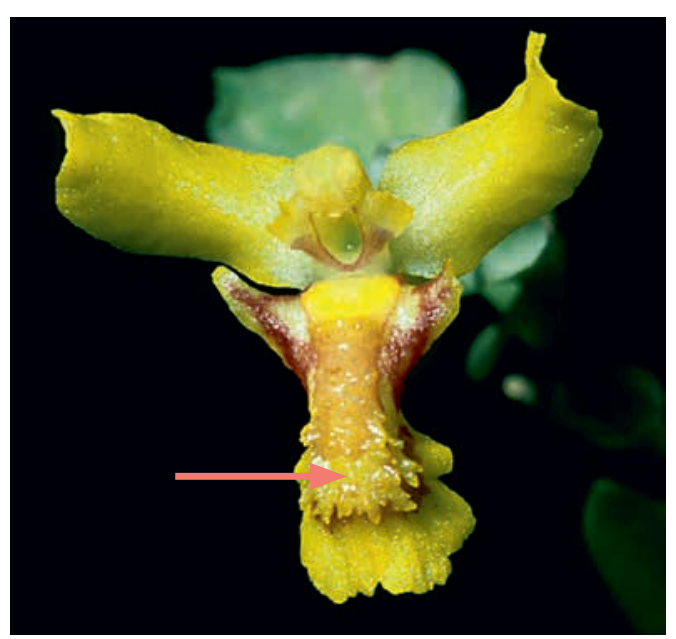

Abb. 11: Blüte von Lockhartia goyazensis (Orchidaceae). Das Labellum trägt einen massiven Kallus, an dessen distalem Ende sich ein Trichom-Elaiophor anschließt (Pfeil)

(Foto: G. Gerlach; Brasilien, Edo. Rôndonia, Cacaulândia; cult. BG München-Nymphenburg, 10/2006).
1959), erkannte aber damals noch nicht, dass sie Öle als Attraktans anbieten. Er beschrieb die „vollständige Zweigängigkeit der Blüten“ bei den „Disperidinae“ (jetzt Coryciinae: Ceratandra, Corycium, Disperis und Pterygodium) und stellte sie in exakten Zeichnungen dar. Nachdem Vogel im ersten Band seiner Ölblumentrilogie (1974) versuchte, die Zweispornigkeit von Diascia (Scrophulariaceae) mit der Ausbeutung eines paarigen Organs analog zu der von ihm beobachteten neotropischen Gattung Angelonia (Plantaginaceae) zu deuten, beobachteten Steiner \& Whitehead (1988, 1990) den Blütenbesuch von Diascia-Arten durch ölsammelnde Bienen der Gattung Rediviva. Ihnen fiel dabei auf, dass die Tiere oft eines oder mehrere Orchideenpollinarien trugen. Da die Morphologie der Pollinarien ein diagnostisches Merkmal der Orchideen-Gattungen ist, folgerten sie, dass die Bienen zuvor Disperis-Blüten besucht haben mussten. So wurde von den beiden südafrikanischen Forschern der Erstnachweis von ölanbietenden Orchideen in der Alten Welt geführt. Dabei werden Disperis-Arten wie die sympatrisch vorkommenden Diascia-Arten von Bienen der Gattung Rediviva bestäubt, indem die Tiere mit ihren verlängerten Vorderbeinen (Zweibeinsammler!) die paarigen Elaiphoren besammeln (STEINER 1989). Heute weiß man, dass fast alle Gattungen/Arten der Subtribus Coryciinae (6 Gattungen, zusammen etwa 120 Arten) Öl als florales Attraktans anbieten und von diversen Arten von Rediviva bestäubt werden. Ausnahmen bilden nur einige Arten von Ceratandra (6 Arten insgesamt), von denen C. grandiflora zur Käferbestäubung übergegangen ist (STEINER 1998).

Ob einige altweltliche Bulbophyllum-, Eriaoder auch neuweltliche Ponthieva-Arten (terrestrisch!) zu den Ölblumen gestellt werden könnten, lässt sich wegen fehlender Beobachtungen nicht sicher sagen. Auf jeden Fall wurden auf den Lippen dieser Pflanzen Ausscheidungen mit öliger Konsistenz gefunden (van der CINGEL 2001). Da aber der sichere Nachweis von fetten Ölen sehr aufwendig ist, lässt sich noch nichts Abschließendes berichten. Das alles zeigt, dass trotz Vogels intensiver Beschäftigung mit Ölblumen der Forschung noch ein weites Betätigungsfeld offen steht. 


\section{Abschließende Bemerkungen zu den Ölblumen}

\subsection{Biogeographie der Ölblumen}

Rückblickend lässt sich sagen, dass uns die Ölblumen in vier Florenreichen entgegen treten: (1) den Neotropen, (2) den Paläotropen, (3) der Capensis (Kapregion Südafrikas) und (4) der Holarktis. Das hinsichtlich der Ölblumen am stärksten differenzierte Florenreich ist das neotropische. Es erstreckt sich über beide amerikanische Subkontinente, von Texas bis nach Patagonien. Als die bedeutendsten Pflanzenfamilien sind die Malpighiaceae, Krameriaceae, Solanaceae, Plantaginaceae, Iridaceae und Orchidaceae zu nennen. Die Bestäuber rekrutieren sich aus zwei Subtriben der Apidae-Apinae: den Centridini (mit den Hauptgattungen Centris und Epicharis) und den Tapinotaspidini (inkl. Tetrapediini). Das Öl wird mit den Vorder- oder zusätzlich mit den Mittelbeinen gesammelt.

Das paläotropische Florenreich erstreckt sich von Westafrika über Madagaskar, Süd- und Südostasien, Neu Guinea bis in den Nordosten Australiens. Bezüglich Ölblumen ist nur eine Familie, die Cucurbitaceae, involviert, und was die Bestäuber betrifft, nur die Gattung Ctenoplectra
(Apidae-Apinae-Ctenoplectrini). Das Öl wird in charakteristischer Weise von den Weibchen mittels einer Bauchbürste („oil-mopping“) aufgesammelt.

Obwohl wesentlich kleiner, ist das Capensische Florenreich hinsichtlich der Ölblumen weitaus stärker differenziert. Als die drei wichtigsten Familien sind hier die Orchideen (insbes. die Subtribus Coryciinae), die Scrophulariaceen (vor allem die Gattung Diascia) und die Stilbaceen (mit Gattungen Anastrabe, Bowkeria und Ixianthes) zu nennen. Als Bestäuber tritt nur die Gattung Rediviva aus der Familie Melittidae auf. Das Öl wird nur mit den Vorderbeinen gesammelt.

Die Holarktis (Nord-Amerika und Eurasien) ist das hinsichtlich der Ölblumen am wenigsten differenzierte Areal. Hier sind die Ölblumen nur auf einen Teil der Gattung Lysimachia (Primulaceae) beschränkt und als Bestäuber ist nur die Gattung Macropis (Melittidae-Melittinae) involviert.

\subsection{Phylogenie der Ölblumen}

Überblickt man die Verteilung der Ölblumen im System der Angiospermen, so fällt auf, dass die Ordnungen, in denen die betreffenden Familien und Gattungen vorkommen, nicht näher mit-

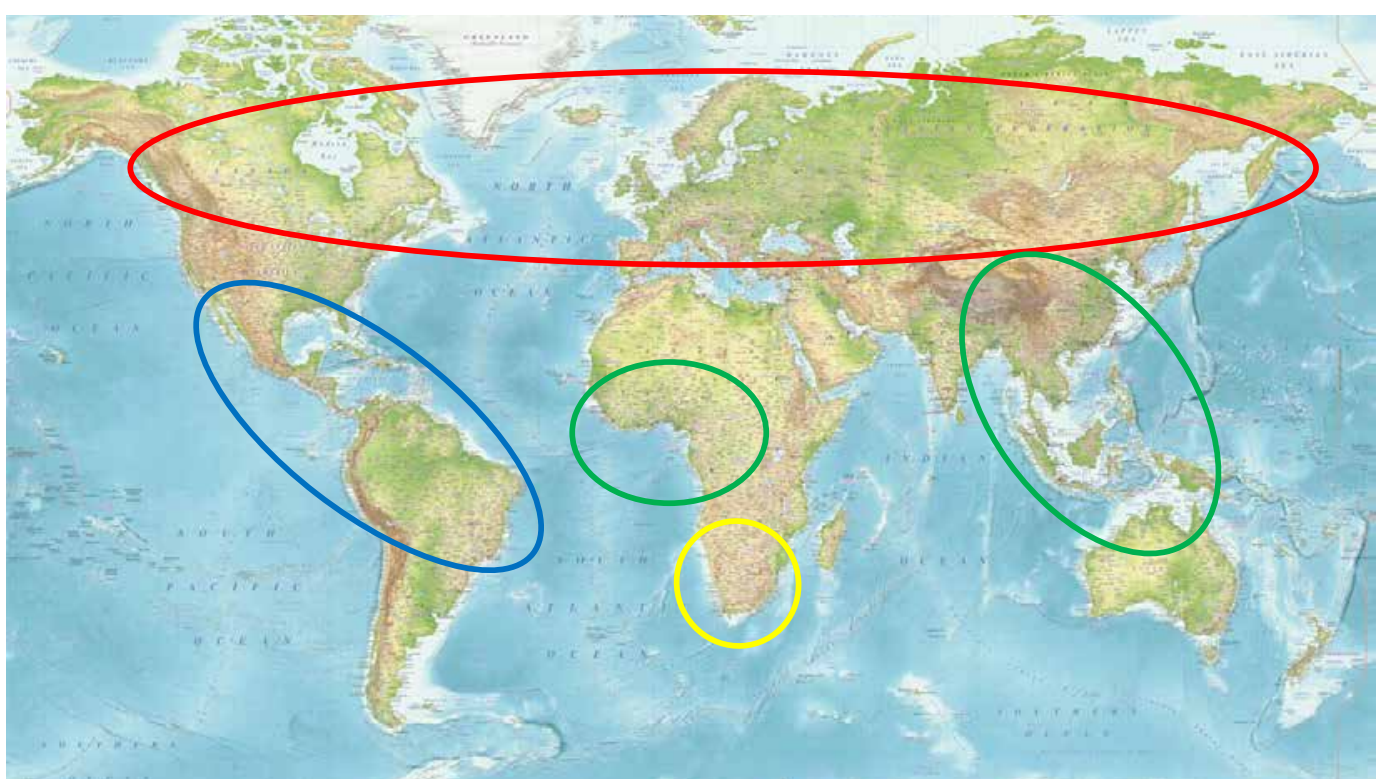

Abb. 12: Grobe geographische Verbreitung der Ölblumen und Ölbienen. Blau: Neotropis, grün: Paläotropis (Cucurbitaceae) Ctenoplectra), gelb: Capensis (Diascia, Disperis/Rediviva), rot: Holarktis (Lysimachia/Macropis). 
einander verwandt sind, und Ölblumen vielfach parallel und voneinander unabhängig entstanden sein müssen. Die Ölblumen können geradezu als Paradebeispiel konvergenter Evolution dienen. Eine Ausnahme scheinen auf den ersten Blick die Lamiales zu machen, in denen mehrere bedeutende Familien mit Ölblumen zu finden sind (Calceolariaceae, Plantaginaceae, Scrophulariaceae und Stilbaceae). Bei näherer Betrachtung stellt man jedoch fest, dass auch hier die Ölblumen vorweisenden Gattungen nicht nächst verwandt sind und deshalb mehrfach unabhängige Evolution viel wahrscheinlicher als ein gemeinsamer Öl-produzierender Vorfahr ist.

Auf Grund des Vorkommens von Ölblumen in 11 Angiospermenfamilien kann man annehmen, dass Ölblumen konvergent mindestens $11 \mathrm{mal}$ entstanden sind. Wahrscheinlicher ist aber, dass Ölblumen sogar wesentlich öfter entstanden sind, sowohl parallel in zwei oder mehr Gattungen einer Familie als auch in mehreren Artengruppen einer Gattung. Wir haben gesehen, dass in vielen Fällen (z. B. Calceolaria, Lysimachia, Momordica, Oncidium etc.) nur ein Teil der Arten einer Gattung ölblütig ist, während die anderen Nektar-, Pollen- oder Täuschblumen darstellen. Das führt zur Frage, ob das Öl in der Evolution manchmal auch wieder verlorengegangen ist.

Diese Frage hat Stefan Vogel mehrfach diskutiert, besonders eingehend im Falle der Malpighiaceae (Vogel 1990). Diese Familie umfasst etwa 1250 Arten in 68 Gattungen, in 250 Arten fehlt das Öl. Letztere sind meist in den Paläotropen (West und Ostafrika, Madagaskar, Arabische Halbinsel, 1 Gattung/15 Arten in Ost- und Südostasien) heimisch. Bei ihnen bilden die Kelchdrüsen kein Öl, sondern Nektar. Sind nun die paläotropischen Arten als eine Reliktgruppe einzustufen, die „noch“ kein Öl bilden, oder ist es umgekehrt? Stammen die paläotropischen Arten von den amerikanischen ab und haben die paläotropischen die Ölproduktion, z.B. mangels geeigneter Bestäuber „eingestellt"?

Vogel kam aus morphologischen Überlegungen zum Schluss, dass erstere Variante zu- trifft, und meint, dass die Malpighiaceen vom westlichen Gondwanaland ihren Ausgang genommen haben und erst nach dem Auseinanderdriften des südamerikanischen und afrikanischen Kontinents die Ölblumen in Südamerika entstanden sind. Die vergleichsweise wenigen Gattungen/Arten der Paläotropen würden demnach eine oder mehrere Reliktgruppen darstellen.

So plausibel diese Interpretation erscheint, so wenig hält sie modernen, molekularsystematischen Untersuchungen stand. Davis \& Anderson (2010) konnten klar zeigen, dass sich die paläotropischen Vertreter der Malpighiaceen von den neotropischen ableiten und in mehreren (mindestens 6), zeitlich gestaffelten Einwanderungswellen Afrika und die östlichen Paläotropen erreicht haben.

Auf breiter Basis sind zuletzt Renner \& SCHAEFER (2010) mit molekular-phylogenetischen-chronologischen Methoden der Frage nachgegangen, wie oft sich bei den Angiospermen Ölblumen entwickelt haben, wie oft sie wieder zu Pollen- oder Nektarblumen rückgebildet wurden, und wann diese Ereignisse stattgefunden haben. Dabei zeigte sich folgendes:

(1) Ölblumen sind in der Evolution mindestens 28 mal in 11 Familien parallel entstanden.

(2) Die Entwicklung der ersten Ölblumen geht bis kurz nach der Wende Kreide/Tertiär zurück.

(3) Als erste entstanden wohl die Ölblumen-Syndrome der Malpighiaceae (vor 75-64 Millionen Jahren), später folgten die Cucurbitaceen und der Gilbweiderich Lysimachia.

(4) Die Ölblumen von Calceolaria, Krameria, Iridaceae und Orchidaceae haben sich als letzte entwickelt und sind höchstens ein paar Millionen Jahre alt.

(5) Das Öl ging aber auch mindestens 36-40 mal als florales Attraktans wieder verloren. 


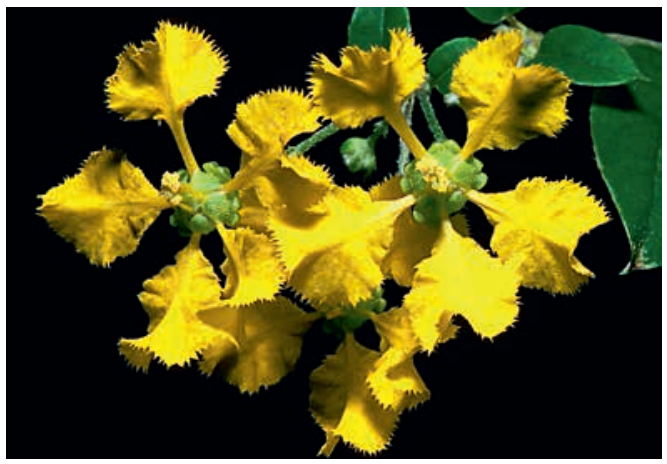

Abb. 13a: Mimikry bei Malpighiaceen und Orchideen. Gaudichaudia mucronata (Malpighiaceae). (Foto: G. GERLACH, BG München-Nymphenburg).

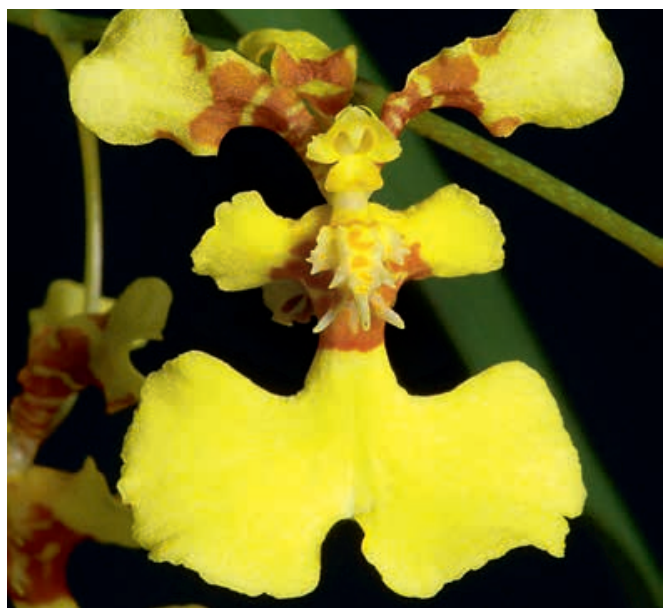

Abb. 13b: Mimikry bei Malpighiaceen und Orchideen. Oncidium hieroglyphicum (Orchidaceae). Näheres im Text. (Foto: G. GerLach, BG München-Nymphenburg).

(6) Dieser Verlust des Öls ging häufig mit der Eroberung neuer Lebensräume einher, in denen geeignete Bestäuber selten waren oder fehlten, so dass Mutanten, bei denen die Öl-Produktion zufällig ausfiel, keinen Bestäubungs-Nachteil hatten und sich langfristig andere Lockstrategien ausbilden mussten.

\subsection{Mimikry}

Im Zusammenhang mit der konvergenten Evolution (Herausbildung ähnlicher Merkmalssyndrome in nicht näher verwandten Gruppen) steht das Phänomen der Mimikry. Im gegenständlichen Fall handelt es sich um Blüten nicht verwandter Gruppen, die Ölblumen größenmäßig, gestalt-

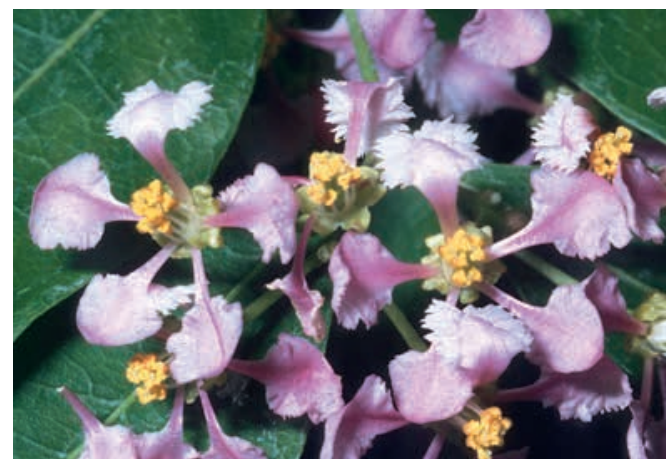

Abb. 14a: Mimikry bei Malpighiaceen und Orchideen. Malpighia glabra (Malpighiaceae). (Foto: G. Gerlach, B.G. München-Nymphenburg).

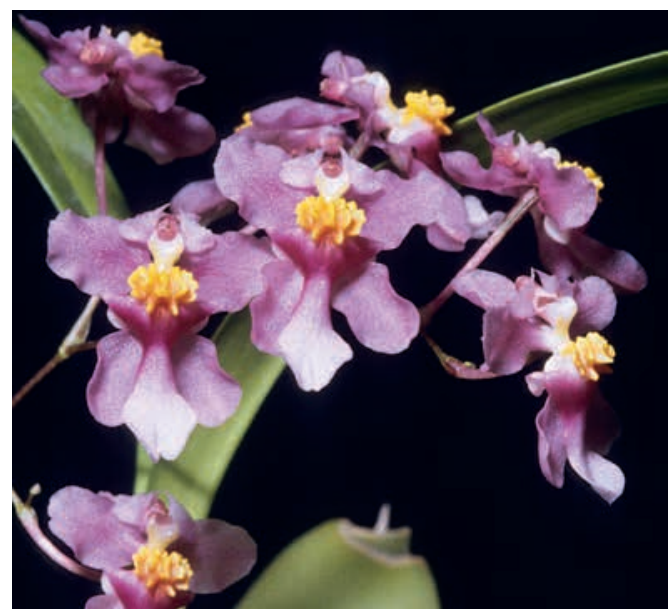

Abb. 14b: Mimikry bei Malpighiaceen und Orchideen. Oncidium sotoanum (Orchidaceae). Näheres im Text (Foto: G. Gerlach, B.G. München-Nymphenburg).

lich und farblich nachahmen (Mimese), sodass sie von Blütenbesuchern nicht oder nur schwer unterschieden werden können. Viele Arten der Orchideengattung Oncidium sind etwa von gleicher Größe, ähnlicher Form und gelber Färbung. Aus der Distanz lässt sich vom Insekt (wie selbst für den Fachbotaniker) nicht ausmachen, ob ein bzw. welches florales Attraktans dargeboten wird. So werden auch jene Blüten, die überhaupt kein Belohnungsmittel bereithalten, bestäubt, indem sie schlichtweg mit solchen verwechselt werden, die eine Belohnung darbieten. Die „unehrlichen“ Blüten leben sozusagen auf Kosten der „ehrlichen“, sie ersparen sich die teure Produktion von Belohnungsmitteln und setzen statt dessen darauf, dass 
sie nicht von den „ehrlichen“ auseinandergehalten werden können.

Noch interessanter sind jene Fälle, in denen imitatorische Konvergenzen bei Blüten verschiedener Pflanzenfamilien auftreten. So ahmen manche Orchideenblüten jene von Malpighiaceae in erstaunlicher Weise nach. Die „ehrlichen“ sind immer die Malpighiaceae, die „unehrlichen“ die Orchideen, die sich das“ Mäntelchen von Ölblumen umhängen“, ohne Öl anzubieten.

Die Entdeckung, dass Ölblumen unseriöse Nachahmer finden, geht, wie könnte es anders sein, ebenfalls auf STEFAN Vogel zurück. Dem Phänomen „Betrug bei Pflanzen“ hat er sogar ein ganzes Büchlein gewidmet (Vogel 1993). Es enthält zahlreiche Beispiele für "Täuschblumen“ und schildert im Detail ihre raffinierten Strategien, Blütenbesucher auszutricksen und hereinzulegen. Es liest sich wie ein Krimi und kann dem Leser wärmstens empfohlen werden.

Besonders spannend wird es bei der Mimikry von Oncidium sotoanum (früher O. ornithorhynchum) und der Blüte mancher Malpighia-Arten (M. glabra, M. emarginata u. a.) die nicht nur in der ungewöhnlichen rosa Färbung, sondern auch in der äußeren Gestalt sehr ähnlich sind, obwohl beide völlig verschiedenen systematischen Gruppen angehören und der Blütenbauplan ganz unterschiedlich ist: Die Malpighiaceen besitzen als typische Vertreter der Dikotyledonen fünfzählige, die Orchidee als Angehörige der Monokotyledonen aber dreizählige Blüten. Während bei den Malpighiaceen die gelben Staubblätter auffällig im Zentrum sitzen, befindet sich bei der Orchidee an dieser Stelle ein gelblich-orangefarbener Kallus. Die Funktion des oberen Blütenblattes, an dem sich die Biene an der Malpighiaceen-Blüte mit ihren Mandibeln festhält, wird bei der Orchidee durch die Säule übernommen, die eine besondere Aussparung zum Einklinken der Mundwerkzeuge besitzt. Auch bei der Orchidee kann der Bestäuber hinter die Blütenebene greifen, und findet dort zwei Elaiophoren (acht bei den Malpighiaceen), die ebenfalls als Epithel-Elaiophoren ausgebildet sind. Durch diese frappanten Ähnlichkeiten, die morphologisch von völlig verschiedenen Blütenorganen herrühren, lässt sich schlüssig erklären, welche Funktion sie tragen. Der Vergleich der Blütendüfte an kultivierten Pflanzen beider Familien ergab ebenfalls Ähnlichkeiten. Aus alldem kann der Schluss gezogen werden, dass hier eine perfekte Müllersche Mimikry vorliegt, bei der die Vorzüge beider Pflanzen geteilt werden, beide belohnen den Bestäuber. Obwohl die beiden Mimikry-Partner keineswegs selten sind, wurden Blütenbesuche bei Oncidium sotoanum bisher noch nie beobachtet. Tatsächlich konnten die Merkmale und die Anpassungen der Blüten so stringent mit konkreten Bestäubungsabläufen erklärt werden, dass Vermutungen mittlerweile schon fast als Tatsachen angenommen werden können.

\subsection{VogELs blütenökologische Studien und Voraussage in der Wissenschaft}

Ein wesentliches Kriterium für das Funktionieren und die Existenzberechtigung der Wissenschaft ist das Kriterium der Voraussagbarkeit. Wenn die Blütenökologie als seriöse Wissenschaft gelten will, dann muss dieses Kriterium zutreffen. Ein spektakuläres Beispiel, das jeder Biologie-Interessierte kennt, ist die Voraussage DARwins über die Bestäubung der madagassischen Orchidee Angraecum sesquipedale: Die großen, weißen und nachts duftenden Blüten dieser Art haben einen über $30 \mathrm{~cm}$ langen, nektarführenden Sporn. DARwin kannte diese Orchidee und postulierte in seinem Orchideen-Buch (DARwin 1862), dass es auf Madagaskar ein Insekt, speziell einen Nachtfalter geben müsse, der einen ebenso langen Rüssel hat. Ein solches Insekt konnte sich damals kaum jemand vorstellen und DARWINs Postulat wurde belächelt. Aber ein solcher Nachtfalter wurde tatsächlich gefunden, 40 Jahre später und lange nach DARwins Tod. Er wurde Xanthopan morganii praedicta, der „Vorausgesagte“, getauft. (Leider stellte sich später heraus, dass die Falter auf Madagaskar doch kein eigenständiges Taxon darstellen, so dass die praedicta-Form wieder aufgegeben wurde).

Eine Voraussage, die fast ebenso spektakulär ist wie jene DARwins, betrifft die Bestäubung der 
zweispornigen Diascia-Arten (Scrophulariaceae) durch Insekten mit paarigen Sammelorganen in Südafrika durch Stefan Vogel.

In seinem ersten Ölblumen-Buch (VogeL 1974) schildert Vogel den Blütenbau, die Lage und die Anatomie der Elaiophoren von Diascia, konnte aber hinsichtlich der Bestäuber keinerlei Angaben machen. Er sprach nur das Postulat aus, dass es auch in Südafrika eine Bienengruppe geben müsse, welche die neotropischen Centridini und Tapinotaspidini in den Paläotropen blütenökologisch vertreten. Es musste sich um Bienen handeln, die mit den Vorderbeinen in die beiden Sporne hineinlangen und das Öl der dort gelegenen Elaiophoren aufsammelten. Etwas später konnte Vogel die Bienengattung Rediviva den Diascien als mögliche Bestäuber zuordnen (Vogel 1984). Ein Problem bereiteten allerdings die Diascien mit langspornigen Blüten (z.B. D. longicornis, mit einer Spornlänge von $25 \mathrm{~mm}$ ). Keine Biene, namentlich keine Rediviva-Art, war bekannt, die so lange Vorderbeine hatte, dass sie die langen Sporne hätte ausbeuten können. Doch VoGEL war überzeugt, dass es ein solches Insekt mit grotesk langen Vorderbeinen geben müsse, ansonsten wären die langspornigen Blüten schon längst ausgestorben. Kaum wurde die Voraussage publik, wurde eine solche Biene gefangen,

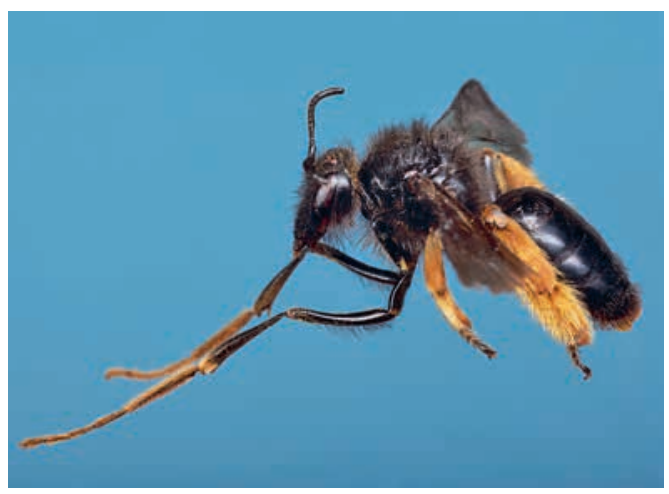

Abb. 15: Rediviva longimanus (Melittidae). Für die Bestäubung der zwei- und extrem langspornigen Blüten einiger Diascia-Arten (Scrophulariaceae) sagte Stefan Vogel die Existenz von Bienen mit extrem verlängerten Vorderbeinen voraus. Diese Voraussage traf durch die Entdeckung zweier neuer Bienenarten (Rediviva longimanus und $R$. emdeorum) mit eben diesen Merkmalen ein (Foto: A. PAuw, Cederberg, Südafrika). und zwar von dem deutschen Botaniker-Ehepaar Ute und Dietrich Müller-Doblies, die in Südafrika arbeiteten. Sie setzen sich sofort mit Vogel in Verbindung, und dieser wandte sich an den amerikanischen Bienen-Spezialisten CHARLES D. Michener. Bald nach der Voraussage wurde die Entdeckungsgeschichte publiziert (VogeL 1984) und die Biene als eine für die Wissenschaft neue Spezies beschrieben (Vogel \& Michener 1985). Sie trägt den Namen Rediviva emdeorum (das Epitheton emdeorum nimmt etwas kryptisch Bezug auf die Entdecker der Biene:. „Em“ und „de“ stehen für die mündliche Aussprache der Initialen M[ÜLLER]-D[oblies) und der Genetiv plural weist darauf hin, dass beiden Ehepartnern die Entdeckung zu verdanken ist). Eine zweite Art mit sehr langen Vorderbeinen wurde ebenfalls von Michener beschrieben, sie wurde mit dem bezeichnenden Namen Rediviva longimanus (die „Langhändige“) belegt.

Im Werk Stefan Vogels sind zahlreiche Voraussagen zu finden, wenn auch nicht alle so spektakulär wie im Falle von Diascia sein mögen. Sie harren noch vielfach der Bestätigung, oder Widerlegung, durch gezielte Feld-Studien. Seine Voraussagen bezüglich südafrikanischer Pflanzen (auf Grund der Blütensyndrome, Vogel 1954) hat man jüngst auf den Prüfstand gestellt (JoHnson \& Wester 2017). Sie erwiesen sich hinsichtlich Vogelbestäubung (Ornithophilie) in 93\% (!) der Fälle als zutreffend, Selbst im schlechtesten Fall (Nachtschwärmerbestäubung, Sphingophilie) lag die Trefferquote noch bei fast 70\%. Was speziell die Ölblumen bei den Orchideen betrifft, sei eine weitere Studie des südafrikanisches Forschers Anton Pauw genannt, die den bezeichnenden (übersetzten) Titel trägt „Blütensyndrome sagen exakt die Bestäubung durch eine spezialisierte ölsammelnde Biene (Rediviva peringueyi, Melittidae) in einer Gilde südafrikanischer Orchideen“ voraus (PAuw 2006).

Stefan Vogel hat mit seinen blütenökologischen Untersuchungen nicht nur großartige Entdeckungen gemacht, sondern auch wesentlich dazu beigetragen, die Blütenökologie als eine ernstzunehmende und „exakte“ Wissenschafts- 
disziplin zu etablieren. Die „Ölblumen“ spielen dabei eine besonders wichtige Rolle.

\section{Literatur}

Chase, M. W., Williams, N. H., Neubig, K. M. \& WhitTEN, W. M. 2008: Taxonomic transfers in Oncidiinae to accord with Genera Orchidacearum, vol. 5. - Lindleyana 21: 20-31.

Cingel, N. A. van Der 2001: An atlas of orchid pollinationAmerica, Africa, Asia and Australia. - Rotterdam.

DARWIN, C. R. 1862: On the various contrivances by which British and foreign orchids are fertilised by insects, and on the good effects of intercrossing. - London - Deutsche Übersetzung (durch H. G. Bronn): Über die Einrichtungen zur Befruchtung Britischer und ausländischer Orchideen durch Insekten und über die günstigen Erfolge der Wechselbefruchtung. - Stuttgart: Schweizerbart. Online-Zugang: https://www. biodiversitylibrary.org/item/52809\#page/11/mode/1up

Davis, C. C. \& Anderson, W. R. 2010: A complete generic phylogeny of Malpighiaceae inferred from nucleotide sequence data and morphology. - Amer. J. Bot. 97: 2031-2048.

Dötterl, S., Gerlach, G. \& Weber, A. 2018: Die großen wissenschaftlichen Leistungen von STEFAN Vogel (19252015). Teil 5b. Öl statt Nektar - die Ölblumen (dikotyle Vertreter). - Palmengarten 82 (2): 48-65.

Goldblatt, P. \& Manning, J. C. 2006: Radiation of pollination systems in the Iridaceae of sub-Saharan Africa. - Ann. Bot. 97: 317-344.

Johnson, S. D. \& Wester, P., 2017: Stefan Vogel's analysis of floral syndromes in the South African flora: an appraisal based on 60 years of pollination studies. - Flora 232: 200-206.

Manning, J. C. \& Goldblatt, P. 2005: Radiation of pollination systems in the Cape genus Tritoniopsis (Iridaceae: Crocoideae) and the development of bimodal pollination strategies. - Int. J. Plant Sci. 166:459-474.

Manning, J. C., Goldblatt, P. \& Friss, I. 2002: The pollination of Tritoniopsis parviflora (Iridaceae) by the oil-collecting bee Rediviva gigas (Hymenoptera: Melittidae): the first record of oil-secretion in African Iridaceae. - South African J. Bot. 68: $171-176$

Neubig, K. M., Whitten, W. M., Williams, N., Blanco, M. A. Endara, L., Burleigh, J. G., Silvera, K., Cushman, J. C. \& Chase, M. W. 2012: Generic recircumscriptions of Oncidiinae (Orchidaceae: Cymbidieae) based on maximum likelihood analysis of combined DNA datasets. - Bot. J. Linn. Soc. 168: 117-146.

PAuw, A. 2006: Floral syndromes accurately predict pollination by a specialized oil-collecting bee (Rediviva peringueyi, Melittidae) in a guild of South African orchids. - Amer. J. Bot. 93: 917-926.
Pijl, L. van der \& Dodson, C. H. 1966: Orchid flowers: their pollination and evolution. -Coral Gables, FL.

Renner, S. S. \& Schaefer, H. 2010. The evolution and loss of oil-offering flowers - new insights from dated phylogenies for angiosperms and bees. - Phil. Trans. Roy. Soc. B 365: 423-135.

Steiner, K. E. \& Whitehead, V. B. 1990: Pollinator adaptation to oil-secreting flowers - Rediviva and Diascia. - Evolution 44: 1701-1707.

Steiner, K. E. 1989: The pollination of Disperis (Orchidaceae) by oil-collecting bees in southern Africa. - Lindleyana 4: 164-183,

Steiner, K. E. 1998: The evolution of beetle pollination in a South African orchid. - Amer. J. Bot. 85: 1180-1193.

Steiner, K. E. \& Whitehead, V. B. 1988: The association between oil-producing flowers and oil-collecting bees in the Drakensberg of Southern Africa. - Monogr. Syst. Bot. Missouri Bot. Gard. 25: 259-277.

Vogel, S. 1954: Blütenbiologische Typen als Elemente der Sippengliederung, dargestellt anhand der Flora Südafrikas. Bot. Studien, Heft 1: 1-338. Jena.

Vogel, S. 1974: Ölblumen und ölsammelnde Bienen. - Abh. Akad. Wiss. Lit. Mainz, Math.-Naturwiss. Kl. 7: 285-547. Wiesbaden.

Vogel, S. 1984: The Diascia flower and its bee: an oil-based symbiosis in Southern Africa. - Acta Bot. Neerl. 33: 509-518.

Vogel, S. 1990: History of the Malpighiaceae in the light of pollination ecology. - Mem. New York Bot. Gard. 55: 130-142.

Vogel, S. 1993: Betrug bei Pflanzen: Die Täuschblumen. Abh. Akad. Wiss. Lit. Mainz, Math.-Naturwiss. Kl. 1993: $1-48$.

Vogel, S. \& Michener, C. D. (1985): Long bee legs and oil producing floral spurs, and a new Rediviva (Hymenoptera, Melittidae; Scrophulariaceae). - J. Kansas Entomol. Soc. 58: 359-364.

\section{Anschriften der Autoren}

Prof. i. R. Dr. Anton Weber, Universität Wien, Department für Botanik und Biodiversitätsforschung, Rennweg 14, A-1030 Wien, E-Mail: anton.weber@univie.ac.at

Dr. Günter Gerlach, Botanischer Garten MünchenNymphenburg, Staatliche Naturwissenschaftliche Sammlungen Bayerns (SNSB), Menzinger Str. 61, 80638 München, E-Mail: gerlach@snsb.de

Prof. Dr. Hanno Schaefer, Technische Universität München, Wissenschaftszentrum Weihenstephan für Ernährung, Landnutzung und Umwelt, Department Ökologie und Ökosystem Management, Biodiversität der Pflanzen, Emil-Ramann-Straße 2, 85354 Freising, E-Mail: hanno.schaefer@tum.de 